\title{
Lacunar stroke in COVID-19
}

\section{Dinesh Chouksey, Pankaj Rathi, Rishu Garg, Rohit Prabhash}

Department of Neurology, SAMC and PGI, Indore, India

The SARS-CoV-2 infected cases are increasing day by day, and there are approximately 80 million cases in the first week of January 2021 worldwide. Recently we all know in COVID-19, about 5-6\% incidence of stroke reported in the two most extensive studies [1,2]. Ischemic strokes are most common, and this might be due to prothrombotic state linked to SARS-CoV-2 virus. The mechanism and management protocol of stroke in COVID patients is still evolving. Till date as per best of our knowledge, most of the reported cases are due to large vessel involvement, and it is worth to discuss the mechanism of small vessel lacunar stroke in such patients. We describe the clinical presentation, radiological features, laboratory parameters and outcome of COVID-19 patient.

A 60 yrs old female, who lost her husband just 02 days before because of COVID-19 disease, admitted with fever, myalgia, throat pain and cough since 3-4 days and her nasal and throat swab RT PCR test positive for SARS-CoV-2 virus. The patient developed sudden onset weakness of left upper and lower limb on the 4th day of her admission to the hospital. She also developed slurring of speech and difficulty in swallowing with flattening of the left nasolabial fold. She is having a history of well-controlled hypertension for 05 years on medicine. Her NIHSS score was 09 at onset and no respiratory distress. CT head plain was done, which showed hypodensity at right corona radiata. The MR angiography brain was done, which shows the right corona radiata diffusion restriction suggestive of lacunar infarct and normal large vessels. Her blood investigation shows normal total leucocyte and platelets count. Her ESR was $14 \mathrm{~mm} / \mathrm{hr}$, CRP $<0.6$ (n $<0.6 \mathrm{mg} / \mathrm{dl})$, D dimer high $600 \mathrm{mg} / \mathrm{l}$ (n < 250), ferritin high 369.9 ng/1 (n 10-291). Her ECG, ECHO and X-ray chest was normal. The patient has treated with Aspirin $150 \mathrm{mg}$ with statin and Azithromycin $500 \mathrm{mg}$ daily, Hydroxychloroquine $400 \mathrm{mg}$ per day and RT feeding. She remained stable, and her weakness improved to 1-grade MRC in both upper limb and lower limbs gradually and able to swallow over the next two weeks. At 20th day of admission, her COVID-19 sample becomes negative, and she has discharged at 30th day of admission.

Lacunar strokes are usually an acute presentation of the ongoing chronic process and less disabling physically but not benign and 30\% left dependent [3]. There are several mechanisms by which lacunar stroke can occur like lipohyalinosis or fibrinoid necrosis, vasospasm, microemboli, arteriolar endothelial dysfunction. The chronic small vessel disease (SVD) changes of white matter due to lipohyalinosis have not seen in our patient MRI, and neither vasospasm changes have seen. The microemboli causing small vessel ischemia leading to acute lacunar stroke in our patient could be possible due to prothrombotic state linked to SARS-CoV-2 virus $[4,5]$. Several recent studies show higher D dimer and ferritin levels in COVID-19 patients which confirm the hypercoagulable state of these patients. Lacunar stroke by arteriolar endothelial dysfunction is the most acceptable mechanism. The vascular endothelium located between blood and brain tissue and the balance between endothelial-derived coagulation fibrinolysis factors, of pro-and anti-inflammation, is essential for vascular homeostasis [6]. The SARS-CoV-2 virus enters the host by using the angiotensin-converting enzyme 2 (ACE2) receptors. These receptors are 


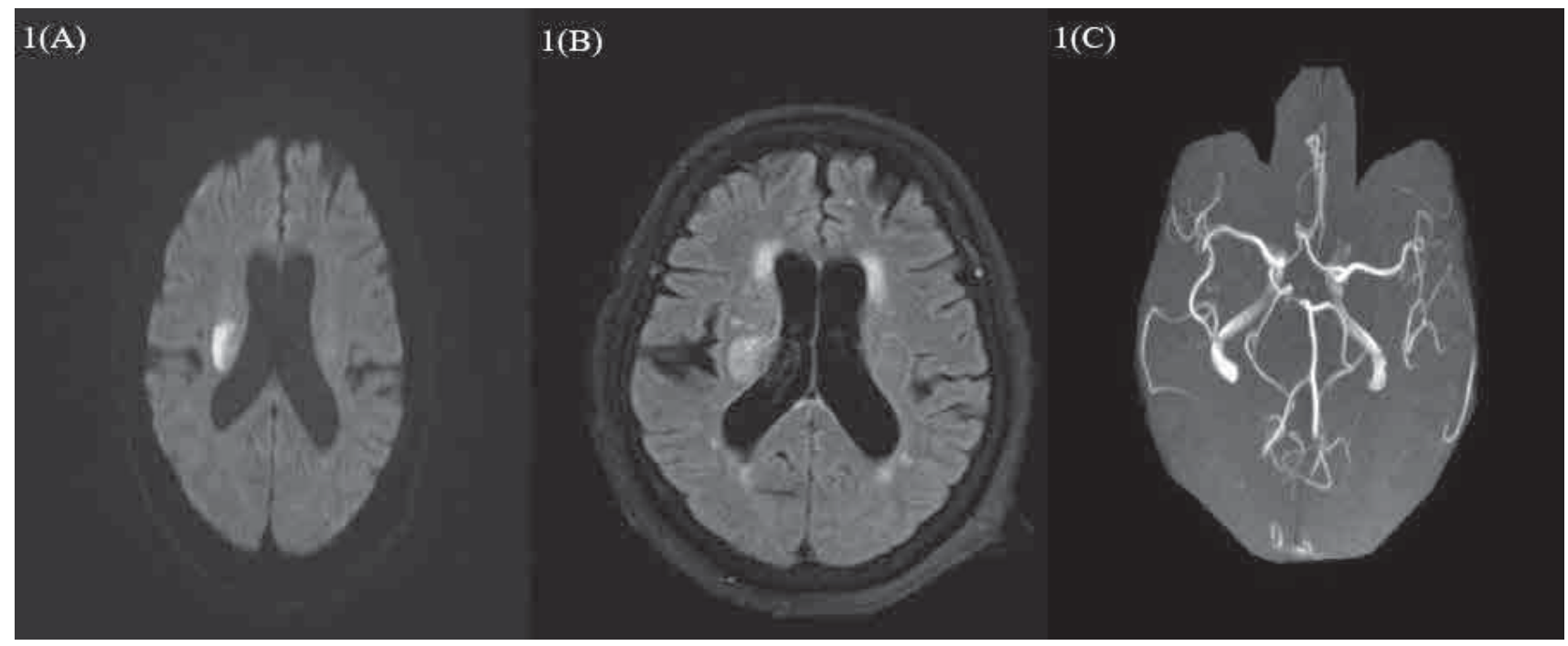

FIGURE 1. 1(a) MRI brain diffusion image shows diffusion restriction at right corona radiata; 1 (b) FLAIR; 1 (c) Angiography shows normal vessels

found in the lung, heart, kidney, intestine and widespread throughout the brain (especially at the cardio-respiratory centre of the brain stem, motor cortex, raphe) and vascular endothelium [7,8]. There is evidence of direct viral infection of the endothelial cell, and diffuse endothelial inflammation results in widespread endothelial dysfunction leading to ischemia and tissue infarct [7]. Recently virus identified in postmortem sample of the brain in frontal lobe inside the neural cell body, vascular endothelium suggests breach in blood-brain barrier directly by a virus [9]. Inflammation may be associated not only with large-vessel atherosclerosis but also with the cerebral small-vessel disease. In our patient no signs of CNS angiitis (stenosis and dilatation of vessel 'beading appearance') seen in MR angiography neither abnormal laboratory parameters of systemic inflammation like CRP and ESR, those were normal. A study by Naser Mobarra et al. concluded that the level of CRP at admis-

Conflict of interest: none declared

Financial support: none declared

\section{REFERENCES}

1. Mao L, Jin H, Wang M, et al. Neurologic Manifestations of Hospitalized Patients With Coronavirus Disease 2019 in Wuhan, China. JAMA Neurol. 2020;e201127.

2. Filatov A, Sharma P, Hindi F, Espinosa PS. Neurological Complications of Coronavirus Disease (COVID-19): Encephalopathy. Cureus. 2020;12(3):e7352.

3. Wardlaw JM. What causes lacunar stroke?. J Neurol Neurosurg Psychiatry. 2005;76(5):617-619.

4. Chen N, Zhou M, Dong X, et al. Epidemiological and clinical characteristics of 99 cases of 2019 novel coronavirus pneumonia in Wuhan, China: a descriptive study. Lancet. 2020;395:507-13.

5. Tang N, Li D, Wang X, et al. Abnormal coagulation parameters are associated with poor prognosis in patients with novel coronavirus pneumonia. J Thromb Haemost 2020;18:844-7. sion might have a clinical implication to identify those at a higher risk of death or recurrence [10]. Our patient has some of the unique features as compared to recently described cases of COVID-19 with stroke, like small vessel involvement (lacunar stroke), low CRP and normal lymphocytes, and patient condition not very sick and had a stroke at the eighth day of the first symptom of COVID-19.

To conclude, SARS-CoV-2 virus association with lacunar stroke in our patient is probably by causing small vessel endothelial dysfunction, and this gives insight towards further research on endothelial cell stabilizing drugs. The use of prophylactic antiplatelets, anticoagulants and statin in the patients of COVID-19 with one of the modifiable risk factors of stroke, as primary prevention, needs to be studied further.

Keywords: lacunar stroke, COVID-19, SARS-CoV-2, stroke in COVID-19
6. Poggesi A, Pasi M, Pescini F, Pantoni L, Inzitari D. Circulating biologic markers of endothelial dysfunction in cerebral small vessel disease: A review. J Cereb Blood Flow Metab. 2016;36(1):72-94.

7. Varga Z, Flammer AJ, Steiger P, et al. Endothelial cell infection and endotheliitis in COVID-19. Lancet. 2020;395(10234):1417-1418.

8. Xia H, Lazartigues E. Angiotensin-converting enzyme 2 in the brain: properties and future directions. J Neurochem. 2008; 107(6):1482-1494.

9. Paniz-Mondolfi A, Bryce C, Grimes Z, et al. Central Nervous System Involvement by Severe Acute Respiratory Syndrome Coronavirus-2 (SARS-CoV-2). J Med Virol. 2020;10.1002/jmv.25915.

10. Mobarra N, Morovatdar N, Di Napoli M, et al. The Association between Inflammatory Markers in the Acute Phase of Stroke and Long-Term Stroke Outcomes: Evidence from a Population-Based Study of Stroke. Neuroepidemiology. 2019;53(1-2):20-26. 